\title{
REGISTRATION OPTIMIZATION OF MOBILE HANDHELD SCANNER POINT CLOUDS WITH STATIC SCANS
}

\author{
V. Alteirac ${ }^{1}$, H. Macher ${ }^{* 1}$, T. Landes ${ }^{1}$ \\ ${ }^{1}$ ICube Laboratory UMR 7357, University of Strasbourg, CNRS, INSA Strasbourg, France (victor.alteirac, helene.macher, \\ tania.landes)@insa-strasbourg.fr
}

KEY WORDS: mobile mapping system, terrestrial laser scanner, point clouds, registration, accuracy, indoor survey, data fusion.

\begin{abstract}
:
In recent years, 3D acquisition methods involving different types of scanners have undergone a phenomenal technological growth. Nowadays, mobile acquisition devices are popular because of their ease of use and their fairly competitive cost. Static scanners provide higher accuracy and more detail, but the acquisition time required with these systems is higher than with mobile systems. Mobile scanners are known for their high acquisition speed but lower point density and accuracy. Until now, the choice of the type of system to use was dependent on the geometry of the study area and the required accuracy. This research aims to find a way to optimize the survey by finding a compromise between the two types of devices, in order to take advantage of both systems for the same acquisition campaign. The first objective is to study the minimum number of static positions required for respecting the required accuracy. A solution is also proposed for compensating the drift of the mobile device. Secondly, the pertinence to use static stations for the principal loop and mobile system for adjoining rooms is investigated. The datasets chosen allow, on the one side, to quantify the limits of the mobile system for the acquisition of indoor buildings and, on the other side, to give recommendations regarding the configuration of static stations as a reference for mobile point clouds. Based on these experiments, a methodology is proposed for indoor environments to combine the use of the two acquisition systems and thus to save time in the field while still providing a good registration quality.
\end{abstract}

\section{INTRODUCTION}

Nowadays, 3D models play an important role particularly in the field of Building Information Modelling (BIM) and in the documentation of cultural heritage. To obtain dense 3D data, laser scanning is the most reliable and accurate solution for indoor modeling. Two types of acquisition systems are used for this purpose: mobile terrestrial mapping systems (MMS) and static terrestrial laser scanners (SLS). These two types of system are generally used separately, although the data they produce might be complementary. This study seeks to find a methodology to take benefit of the use of both systems and to combine data acquired by the two devices. The study will focus exclusively on indoor surveys, captured with a handheld mobile system and a static terrestrial laser scanner.

Each system has advantages and drawbacks. The handheld scanner (GeoSLAM ZEB REVO RT) allows fast, handy and easy acquisition. However, this scanner sometimes produces unreliable data. Indeed, experiments show that the SLAM technology on which the system is based may cause some drift issues in linear surveys. Moreover, the low density and noisy point cloud compared to the static system prevent the use of ground control points for the registration and georeferencing steps. On the other hand, the static laser scanner (FARO Focus 3D X330) provides data with a better density of points and with less noise, but with longer acquisition time. The device is therefore not as easy to use as the other system. Furthermore, it requires traveling with a tripod and targets if we want to produce reliable and georeferenced point clouds.

\footnotetext{
Corresponding author
}

Aim of this study is to develop a methodology by taking benefit of the mobile system without losing accuracy. The main idea is to use SLS stations as ground control static stations for creating a robust network, on which MMS clouds will be registered. The higher quality and georeferenced $3 \mathrm{D}$ point cloud of the FARO Focus 3D will be used to fix the drift of the ZEB REVO RT and simultaneously to georeference the point clouds. This allows combining the acquisition speed of the mobile device with the accuracy of a static scanner. In previous work, the accuracy, noise and limitations of the ZEB REVO RT were assessed (Salgues et al., 2020). This article aims to propose a more specific use of the handheld system, namely coupled with static acquisition stations. This allows an optimal and combined survey with a solid supporting network given by the static scans and a fast acquisition time offered by the mobile system.

\section{RELATED WORK}

Previous studies highlight the fact that the ZEB REVO RT provides a satisfying accuracy by using the SLAM technology (Simultaneous Localization and Mapping). For instance, Nocerino et al. (2017) and Salgues et al. (2020) made experiments in order to estimate the accuracy of the survey and the noisy data produced with the device. They compared directly point clouds acquired with both devices (mobile against static laser scanners) over the same area and reached an accuracy of 3 $\mathrm{cm}$ in compliance with the conditions of use suggested by the manufacturer (GeoSLAM).

Only a few papers deal with the topic of combined registration of handheld mobile and static point clouds. In order to merge both 
types of data, Previtali et al. (2020) explained that a good distribution of static scanners stations along the mobile acquisition trajectory may help to spread the drifting error. For each mobile acquisition dataset, registration with static scanner point clouds is thereafter made. Lachat et al. (2018) suggested to perform a manual registration with common points to align the two point clouds followed by an adjustment using the generalized least squares method. In our study, an acquisition and also a processing methodology is sought.

A robust comparison method between two point clouds is needed. The study of Toschi et al. (2015) presents a two steps comparison of point clouds acquired by different devices. First, an ICP (Iterative Closest Point) algorithm is launched in order to register one on the other. Following this, an M3C2 (Multiscale Model to Model Cloud Comparison) distance calculation is performed for quantifying the differences between the clouds (Lague et al., 2013). This algorithm allows the computation of signed distances between two point clouds. Moreover, by defining appropriate parameters, it enables the filtering of unwanted artifacts in the two point clouds. Once the distances are calculated, a statistical study is carried out. The parameters presented in (1) are calculated to determine if the histogram follows a normal distribution.

Skewness $=\frac{\sum\left(x_{i}-\mu\right)^{3}}{(n-1) \sigma^{3}}$ and Kurtosis $=\frac{\sum\left(x_{i}-\mu\right)^{4}}{(n-1) \sigma^{4}}$

The skewness parameter is related to the asymmetry of the histogram; the value obtained must be as close as possible to 0 . The kurtosis parameter shows whether the histogram of the data has too flat or too hollow areas. This value should be around 3 (Toschi et al., 2015). If the distribution does not follow a normal distribution, a non-parametric model based on the median of the data and the median absolute difference is used. This methodology is based on statistical methods and a robust nonparametric model.

Both statistical parameters will be used to assess the proposed methodology. After a short description of the systems and datasets (section 3), the developed comparison workflow and the results obtained after its application on the datasets will be described (section 4).

\section{ACQUISITION SYSTEMS AND DATASETS}

\subsection{Static and mobile acquisition systems used}

In order to conduct the study, two devices were used: the static laser scanner FARO FOCUS X330 and the handheld mobile mapping system GeoSLAM ZEB REVO RT. The characteristics of both devices are listed in Table 1. In the next sections, the mobile mapping system will be called "MMS" and the static laser scanning system "SLS".

The MMS is composed of a "head" scanner for measurement and an inertial unit for direct positioning. The scanner is connected with an embedded computer that stores and synchronizes the data in real time. It operates using the SLAM technology. This process makes it possible to obtain a 3D representation of an environment and simultaneously to locate oneself in it.

\begin{tabular}{|c|c|c|}
\hline Name & $\begin{array}{ll}\text { FARO } & \text { FOCUS } \\
\text { X330 } & \\
\text { Static } & \text { scanner } \\
\text { (SLS) } & \\
\end{array}$ & $\begin{array}{l}\text { GeoSLAM ZEB } \\
\text { REVO RT } \\
\text { Mobile system } \\
(\text { MMS) }\end{array}$ \\
\hline \multicolumn{3}{|l|}{ Picture } \\
\hline Weight & $5.2 \mathrm{Kg}$ & $850 \mathrm{~g}$ \\
\hline Range & $0.3-300 \mathrm{~m}$ & $30 \mathrm{~m}$ \\
\hline Absolute Accuracy & $2 \mathrm{~mm}$ & $3 \mathrm{~cm}$ \\
\hline Horizontal field of view & $360^{\circ}$ & $270^{\circ}$ \\
\hline Vertical field of view & $300^{\circ}$ & $270^{\circ}$ \\
\hline Resolution & $0.009^{\circ}$ & $\begin{array}{c}0.625^{\circ}(\mathrm{Hz})- \\
1.8^{\circ}(\mathrm{V})\end{array}$ \\
\hline Pts/sec & 976000 & 43000 \\
\hline
\end{tabular}

Table 1. Characteristics of the acquisition systems studied (according to the respective manufacturers)

As already mentioned, unlike the FARO FOCUS X330, the ZEB REVO RT allows faster acquisitions and is easier to use. However, the latter has a lower absolute accuracy, works at shorter ranges and provides point clouds with low density and without RGB information.

\subsection{Study areas and datasets}

In this work, two datasets are considered: SLS and MMS point clouds acquired in the Zoological Museum of Strasbourg and in several rooms of the graduate school INSA Strasbourg (France).

This first dataset, the Zoological Museum, was chosen to test the robustness of the MMS against the SLS, along large loops. Indeed, the Zoological Museum is a building of about $50 \mathrm{~m} \times 50 \mathrm{~m}$ constructed around an interior courtyard. This specific architecture allows to acquire easily horizontal loops (along each floor) and vertical loops (on the way to another floor). The horizontal loop is interesting because it is wide (about $140 \mathrm{~m}$ ) and therefore the drift of the MMS will be easier to study. The vertical loop has been acquired in a stairwell of $18 \mathrm{~m}$ height. This enables to study the drift in Z. The first step will allow to quantify the loss of accuracy when reducing the number of static stations on which the mobile point clouds are registered. Then, in a second step, a solution to handle the drift observed in mobile acquisitions is proposed.

The second dataset consists in several point clouds acquired in the INSA Strasbourg building. Many classrooms are adjoining and overlook the corridor. This dataset has been constructed to test the possibility to merge individual loops obtained in independent rooms with a principal acquisition of the SLS. In this regard, the static acquisitions act as a reference network. 
3.2.1 Dataset 1: Zoological Museum of Strasbourg: Two point clouds were acquired in the museum. They consist of loops acquired in the same area with the handheld system and with the static system. A first loop was acquired in a horizontal plane, along a corridor. In parallel, 30 static stations were acquired along the same trajectory. The second loop is vertical and located in a staircase where 15 static scans were carried out. Figure 1a presents the trajectory of the horizontal loop and Figure $1 \mathrm{~b}$ the vertical loop.

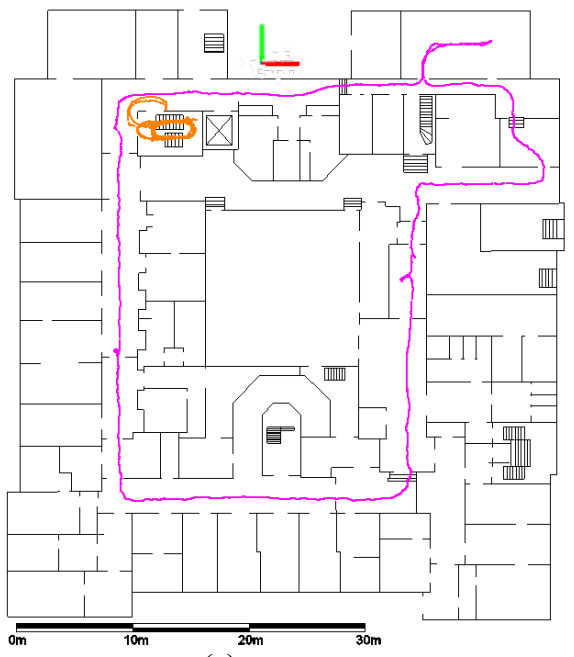

(a)

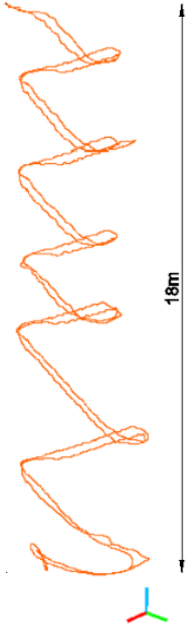

(b)
Figure 1. Trajectories of two loops with the MMS: horizontal loop in pink and vertical one in orange (a), Trajectory of the vertical loop (b). The static stations are not represented.

3.2.2 Dataset 2: INSA Strasbourg building: For this dataset, two point clouds were acquired with the MMS in a corridor and several classrooms. In parallel, 9 SLS stations were carried out to cover the same area. Figure 2 shows the SLS stations, the trajectories (loops1 is about $43 \mathrm{~m}$ and loop2 is about $74 \mathrm{~m}$ long) of the handheld scanner as well as an horizontal slice of the MMS point cloud.

\section{METHODOLOGY AND RESULTS}

In order to analyse the best way to combine MMS and SLS acquisitions, a workflow has been established.

\subsection{Developed workflow for analysing the combinations}

Before performing registrations and comparisons between point clouds, it is necessary to remove inconsistencies between the point clouds to compare, i.e. parts of the point clouds which are not identical in both acquisitions. Indeed, the data from the two systems were not acquired at the same time, so there are objects present in one cloud but not in the other. In addition, the static scanner has a longer range, i.e. points outside the corridor were recorded and are not present in the mobile data. Therefore the SLS data that is not in common with the MMS data were removed, based on a coarse manual registration (Figure 3). This filtering is important because inconsistent point clouds could alter the quality of the ICP registration step. A spatial resampling of one point per centimeter is also carried out in order to reduce the data and therefore the computation time.

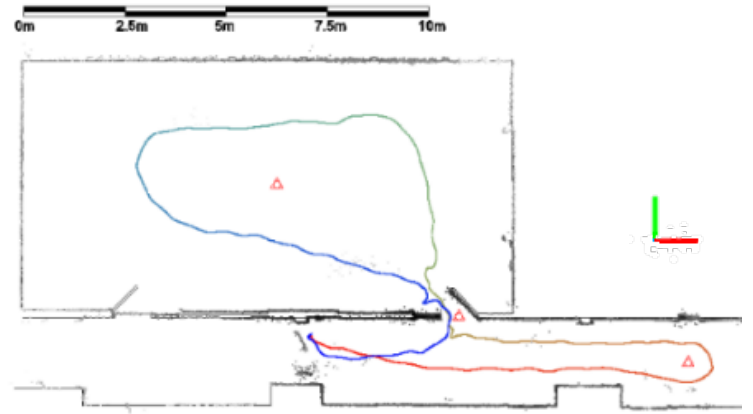

(a)

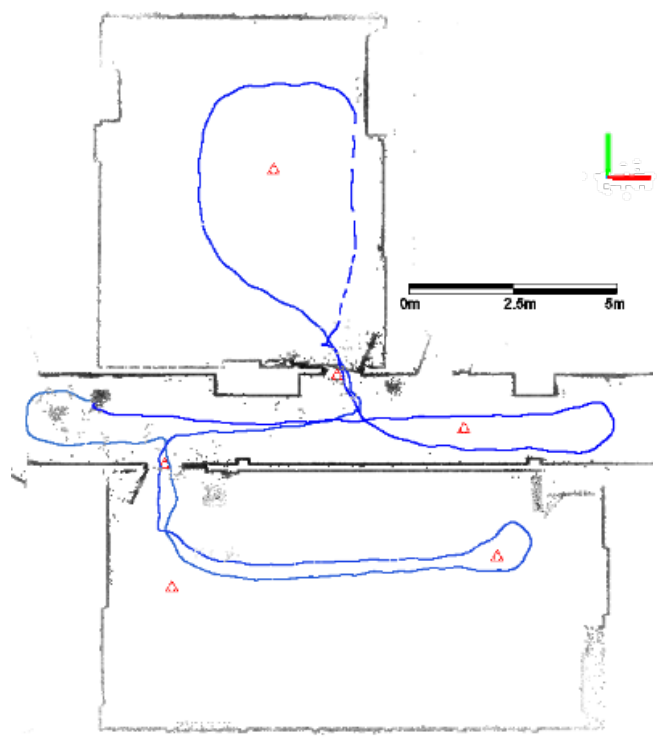

(b)

Figure 2. Distribution of SLS stations (red triangles) superimposed with the point cloud of the MMS acquisition for two loops, in a top view. (a) Loop 1, (b) Loop 2.

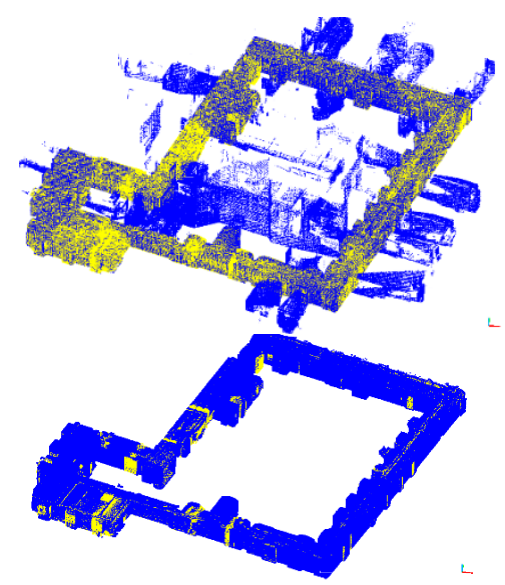

Figure 3. Pre-processing of two datasets, MMS (yellow) and SLS acquisitions (blue). Original point clouds (above) and segmented after removing inconsistencies (below). 
Once the "outliers" are removed, the comparison of the suggested combinations can begin. For that purpose, a workflow has been developed and is described in Figure 4.

In a first experiment, all SLS stations are compared to the MMS loops after registration of both datasets. In a second experiment, the MMS loops are registered to a reduced number of SLS stations.

For each stage, the registration is done in two steps. A first manual coarse registration, followed by the ICP algorithm. The reference results are given by a comparison of the MMS loop with all SLS stations of the same area.

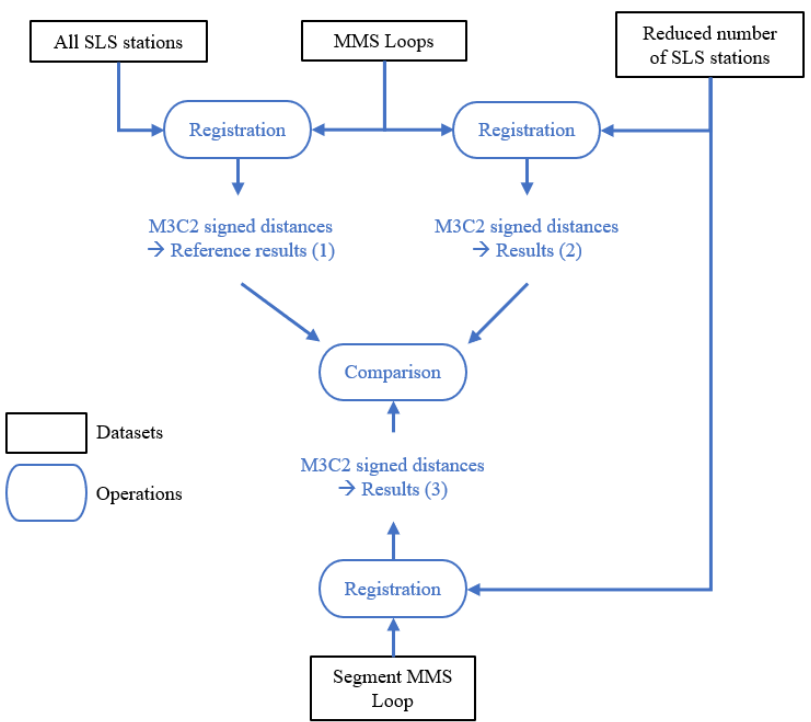

Figure 4. Developed processing chain for the analysis of the combination of both systems.

After a registration step providing a reference result (1), the number of static stations is progressively reduced, and the uncertainty of the registration (2) is analysed. The objective is to find the minimum number of static stations needed to register each loop, i.e. the optimal number of stations to keep.

Since the point clouds provided by the MMS remain almost impossible to refine during a post-processing step, it might be useful to develop a method to exploit the data, that is to correct the deviations caused by SLAM. The solution proposed in this work is to segment the MMS acquisitions and to registrate each segment with a minimum number of static stations (3).

If the results show that a reduction in the number of static scanners and the splitting of the moving point cloud can compensate the drift of the device, a combination of the two methods will be considered.

MMS is very useful for scanning cluttered rooms. For this reason, a test was carried out. It has been decided to scan a complete room with the MMS and to consider a few SLS stations in the corridor. The minimum number and the most appropriate position of the SLS stations in the corridor can therefore be studied. The minimum overlap to be considered between MMS and SLS acquisitions to ensure high accuracy in the room registration is also investigated.

The results of each step are obtained via the analysis of the histogram of signed distances provided by the $\mathrm{M} 3 \mathrm{C} 2$ algorithm. In order to perform these comparisons, the ICP and $\mathrm{M} 3 \mathrm{C} 2$ algorithms are used via the CloudCompare (http://www.cloudcompare.org/ - Version 2.9.1) software. Afterwards, a Matlab script is used to calculate statistical parameters from the signed distances.

\subsection{Application of the processing chain}

4.2.1 Horizontal loop of dataset 1: The first loop studied is the horizontal loop in the basement of the Zoological Museum. The trajectory of the MMS follows a closed corridor with 30 distributed static stations. In addition, a passage through an individual room is made coupled with 2 additional static stations (Figure 1).

In order to register both clouds an ICP algorithm is launched, followed by an $\mathrm{M} 3 \mathrm{C} 2$ distances calculation to compare their deviations. The result of the comparison is presented Figure 5. Additionally, a statistical study is performed with the calculated deviations, as presented in Table 2.

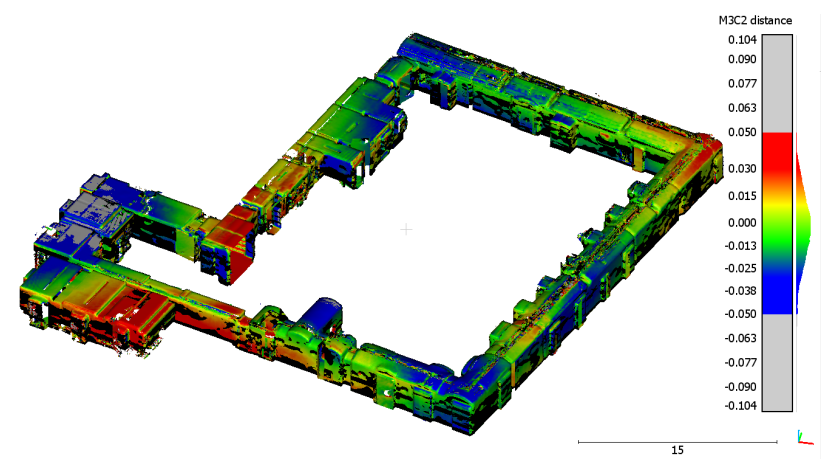

(a)

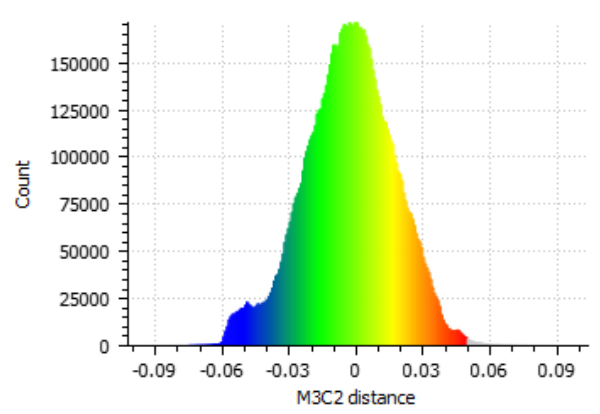

(b)

Figure 5. Result of the comparison with $\mathrm{M} 3 \mathrm{C} 2$ distances (in meters): (a) point cloud colorized according to distances,

(b) associated histogram. 


\begin{tabular}{|c|c|}
\hline Settings & Values \\
\hline Number of static stations & 30 \\
Mean & $-0.4 \mathrm{~cm}$ \\
Standard deviation & $2.1 \mathrm{~cm}$ \\
Median & $-0.4 \mathrm{~cm}$ \\
MAD & $-1.6 \mathrm{~cm}$ \\
Skewness & -0.093 \\
Kurtosis & 3.123 \\
Distances between -3 and $3 \mathrm{~cm}$ & $84 \%$ \\
\hline
\end{tabular}

Table 2. Results of the comparison between point clouds (reference result)

The range $(-3 \mathrm{~cm} ;+3 \mathrm{~cm})$ was chosen regarding the accuracy of the MMS and is therefore considered as reference value. The Skewness and Kurtosis parameters allow to validate that the normal distribution is respected and that it makes sense to use the mean and standard deviation as statistical parameters. The RMS after ICP is $2.1 \mathrm{~cm}$. A standard deviation of $2.1 \mathrm{~cm}$ and a rate of $84 \%$ of distances between -3 and $3 \mathrm{~cm}$ are given. These are socalled "reference results" in Figure 4 for the next comparisons, i.e. with reduced number of SLS stations and with segmented MMS loops.

The first experiment can now be done, which consists in reducing the number of SLS stations. Two levels are considered for the reduction. The first level consists in reducing the number of SLS stations by half (15) so that these stations have enough overlap between them to be directly aligned. The second level consists in reducing the number of stations to 6 but making the assumption that these last ones can be registered via an external technique (total station for instance). There are six corners in this corridor (see Fig. 6), so it has been decided to keep six SLS stations, one per corner, i.e. at the positions where the trajectory of the MMS varies suddenly. Additional intermediate stations between the corners are added for direct georefencing. An ICP algorithm is launched on the MMS data in order to register it with the reduced number of SLS stations. Then M3C2 is applied to calculate the distances separating both clouds.

\begin{tabular}{|c|c|c|}
\hline Settings & \multicolumn{2}{|c|}{ Values } \\
\hline Number of static stations & 15 & 6 \\
Mean & $0.2 \mathrm{~cm}$ & $0.1 \mathrm{~cm}$ \\
Standard deviation & $2.3 \mathrm{~cm}$ & $2.4 \mathrm{~cm}$ \\
Median & $0.1 \mathrm{~cm}$ & $0.3 \mathrm{~cm}$ \\
MAD & $1.8 \mathrm{~cm}$ & $1.9 \mathrm{~cm}$ \\
Skewness & -0.083 & -0.011 \\
Kurtosis & 2.92 & 2.83 \\
Distances between -3 and $3 \mathrm{~cm}$ & $80.4 \%$ & $78.9 \%$ \\
\hline
\end{tabular}

Table 3. Results of registration with 15 and 6 static stations

As shown in Table 3, compared to the reference result, the percentage of distance between -3 and $3 \mathrm{~cm}$ decreases by $4 \%$ for 15 selected stations and by $6 \%$ for 6 stations. In addition, the RMS from the ICP algorithm is $2.1 \mathrm{~cm}$ for 15 stations and $2.3 \mathrm{~cm}$ for 6 stations. There is a slight loss of accuracy. This leads to the conclusion that it is acceptable to reduce the stations and to make this compromise between MMS acquisition and SLS stations for the acquisition of a linear of this type.
Another experiment consists in trying to reduce the drift caused by the SLAM technology. The idea is to segment the trajectory of the MMS acquisition. Even if it does not correct the drift, this solution distributes the errors piecewise along the trajectory. The position of the static stations along the segmented trajectory is illustrated in Figure 6. The statistical results are summarized in Table 4 .

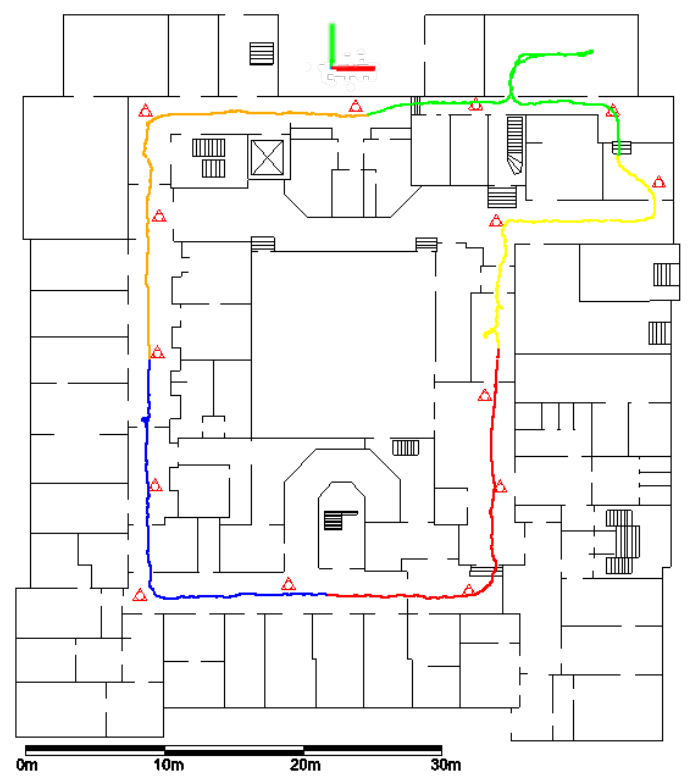

Figure 6. Distribution of static stations (red triangles) and segments of point clouds (on color per segment) of the MMS created along the trajectory.

\begin{tabular}{|c|c|}
\hline Settings & Values \\
\hline Number of static stations & 15 \\
Number of segments & 5 \\
Mean & $-0.3 \mathrm{~cm}$ \\
Standard deviation & $1.7 \mathrm{~cm}$ \\
Median & $-0.4 \mathrm{~cm}$ \\
MAD & $-1.3 \mathrm{~cm}$ \\
Skewness & -0.32 \\
Kurtosis & 3.93 \\
Distances between -3 and $3 \mathrm{~cm}$ & $91.3 \%$ \\
\hline
\end{tabular}

Table 4. Statistical results for MMS cloud segmentation and reduction of the number of SLS stations.

It is now necessary to check that the point clouds are correctly aligned with each other and that there are no breaks between the segments. To do this, the point clouds are sliced in a $5 \mathrm{~cm}$ thick plane to check planimetric and altimetric coherence as shown in Figure 7. The average difference between the slices is $1.5 \mathrm{~cm}$ in $\mathrm{XY}$ and $3.1 \mathrm{~cm}$ in $\mathrm{Z}$. The results obtained in $\mathrm{XY}$ are correct, but it is less satisfactory in Z. Note that the same tests were carried out after reducing the number of static stations to 6 . However, differences between segments of $8.5 \mathrm{~cm}$ appeared, which means an important decrease in accuracy. 

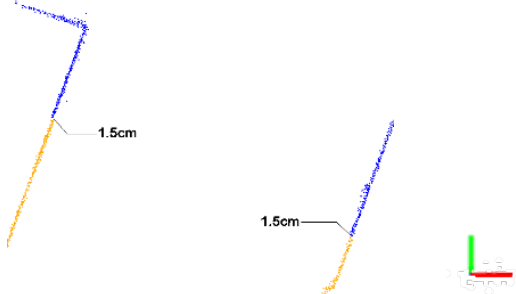

Figure 7. Distances between two segments of point clouds after segmentation (blue and orange points) in $\mathrm{X}, \mathrm{Y}$

4.2.2 Vertical Loop of dataset 1: The processing steps carried out for the vertical loop are similar to those considered for the horizontal loop. After manual registration and segmentation, an RMS of $7 \mathrm{~cm}$ is obtained after ICP. This deviation is too high, and the bad results are confirmed by the M3C2 distances obtained (Figure 8).

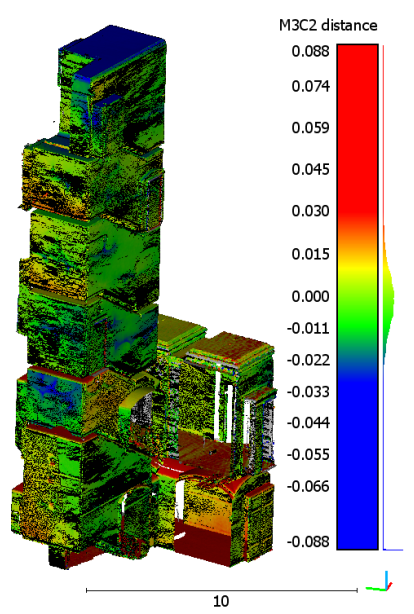

Figure 8. Representation of the $\mathrm{M} 3 \mathrm{C} 2$ distances between the complete MMS acquisition and all SLS stations.

\begin{tabular}{|c|c|}
\hline Settings & Values \\
\hline Number of static stations & 10 \\
Mean & $-0.0 \mathrm{~cm}$ \\
Standard deviation & $1.6 \mathrm{~cm}$ \\
Median & $-0.1 \mathrm{~cm}$ \\
MAD & $-1.2 \mathrm{~cm}$ \\
Skewness & -0.28 \\
Kurtosis & 3.99 \\
Distances between -3 and $3 \mathrm{~cm}$ & $93.0 \%$ \\
\hline
\end{tabular}

Table 5. References results for the vertical loop with all SLS stations and non-segmented MMS loop.

Based on the results presented in Figure 8, it can be noticed that the first (in red) and last landing (in blue) of the staircase present large deviations to the reference. It looks like a compression of the mobile point cloud compared to the static one. It is assumed that the inertial unit has encountered some kind of problem during the acquisition like a sudden movement. The methodology step to segment mobile acquisition should correct or at least reduce this problem. In order to confirm this hypothesis, the mobile point cloud has been segmented and then registered by using all the SLS stations (10 stations). The number of slices has been defined so that one segment per landing of the stairwell is kept according to the scheme in Figure 9.
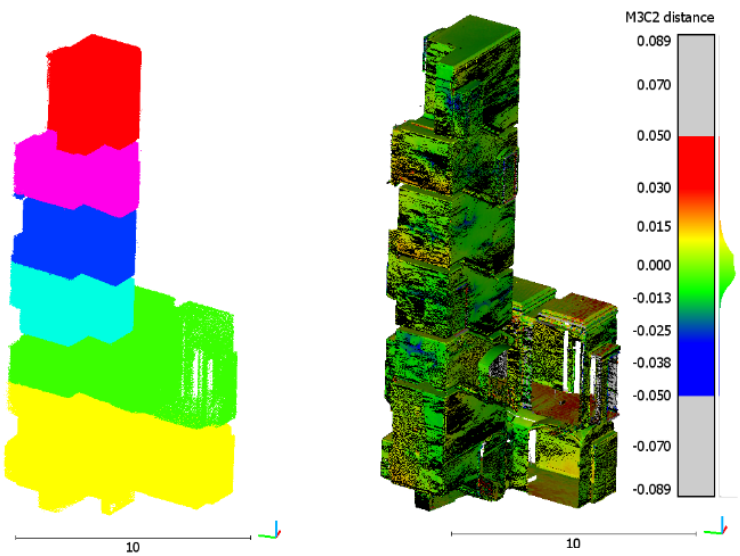

Figure 9. Representation of the MMS segments (left), and $\mathrm{M} 3 \mathrm{C} 2$ results (right).

\begin{tabular}{|c|c|}
\hline Settings & Values \\
\hline Number of static stations & 10 \\
Mean & $-0.1 \mathrm{~cm}$ \\
Standard deviation & $0.9 \mathrm{~cm}$ \\
Median & $-0.0 \mathrm{~cm}$ \\
MAD & $-0.7 \mathrm{~cm}$ \\
Skewness & -0.032 \\
Kurtosis & 9.2 \\
Distances between -3 and $3 \mathrm{~cm}$ & $99.7 \%$ \\
\hline
\end{tabular}

Table 6. Statistical result after segmenting the MMS cloud in 6 sections.

The results are rather satisfactory, the compression effect of the mobile acquisition point cloud seems to be reduced and as a consequence the distance rate between -3 and $3 \mathrm{~cm}$ has increased. The error seems to have been fixed but, as expected, not fully corrected.

After distribution of the errors along the vertical trajectory, a reduction of the number of SLS scans was performed. Only one SLS station is kept per floor in order to keep one station for each segment of the MMS. It is assumed that the stations can be georeferenced via an external method. Seven stations are retained with sufficient coverage and the previous segmentation of MMS acquisition is kept.

\begin{tabular}{|c|c|}
\hline Settings & Values \\
\hline Number of static stations & 7 \\
Mean & $-0.1 \mathrm{~cm}$ \\
Standard deviation & $1.0 \mathrm{~cm}$ \\
Median & $-0.1 \mathrm{~cm}$ \\
MAD & $-0.7 \mathrm{~cm}$ \\
Skewness & -0.09 \\
Kurtosis & 7.5 \\
Distances between -3 and $3 \mathrm{~cm}$ & $99.0 \%$ \\
\hline
\end{tabular}

Table 7. Results after segmenting MMS acquisitions and reducing the number of SLS stations. 
The differences seem similar to those obtained with all SLS stations. This proves that reducing the number of stations does not lead to a great loss of accuracy in this case. Indeed, a decrease of $1 \mathrm{~mm}$ in the standard deviation and $0.7 \%$ of distances between -3 and $3 \mathrm{~cm}$ are observed. As for the horizontal loop, the junctions must be checked between the segments. A deviation of $1.5 \mathrm{~cm}$ is observed on average for all junctions. The total of these gaps is $7.1 \mathrm{~cm}$ which corresponds logically to the reference deviation observed between the mobile and static acquisitions. The methodology consisting in segmenting the point cloud and distributing the errors along the entire stairwell with the MMS is a satisfying solution under the condition to have SLS stations at least on the extremities of the stairwell.

\subsection{Registration of individual rooms}

4.3.1 Isolated room in the Zoological Museum: When the Zoological Museum was scanned, an isolated room was recorded with the MMS and two SLS stations are placed in the corridor. A registration is therefore performed based on the common point clouds. One complementary SLS station has been placed in the room so that it can be considered as a ground truth.

As shown in Figure 10, the MMS trajectory is presented in blue. It goes along the corridor into the cluttered room and leaves the room to finish the route in the corridor where the SLS stations are located. It must be noticed that the MMS trajectory has not been closed in a loop.

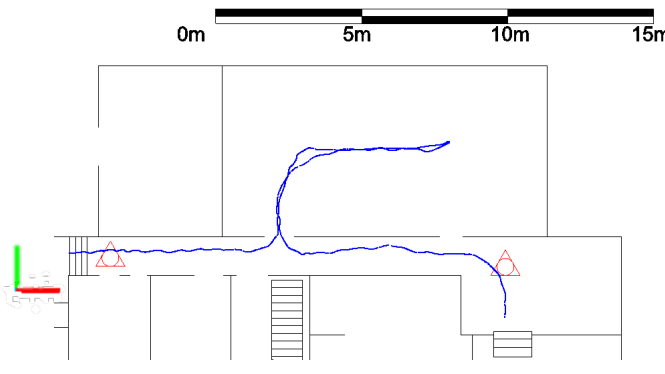

Figure 10. Trajectory of the MMS (in blue), and SLS stations (red triangles)

The MMS point cloud is then registered with the static stations in the corridor. Table 8 presents the results.

\begin{tabular}{|c|c|}
\hline Settings & Values \\
\hline Number of static stations & 2 \\
Mean & $0.1 \mathrm{~cm}$ \\
Standard deviation & $1.4 \mathrm{~cm}$ \\
Median & $-0.1 \mathrm{~cm}$ \\
MAD & $-10 \mathrm{~cm}$ \\
Skewness & -0.108 \\
Kurtosis & 5.7 \\
Distances between -3 and $3 \mathrm{~cm}$ & $96.1 \%$ \\
\hline
\end{tabular}

Table 8. Results for the isolated room

A standard deviation of $1.4 \mathrm{~cm}$ and $96 \%$ of distances located between -3 and $3 \mathrm{~cm}$ prove that the registration of an isolated room can be envisaged to reduce the acquisition time. In order to confirm this result, more detailed experiments were performed in the INSA Strasbourg building, but this time by respecting loops.
4.3.2 First Room of INSA (Loop 1): The distribution of the SLS stations is done according to Figure 2(a). Among the three SLS stations, only two are kept for ICP registration: the first one is located in the corridor and the second one in the door frame. The third station in the room is therefore considered as ground truth and will be used to check the quality of the registration. The results of the comparison with the ground truth are illustrated in Figure 11 and the calculated parameters are listed in Table 9.

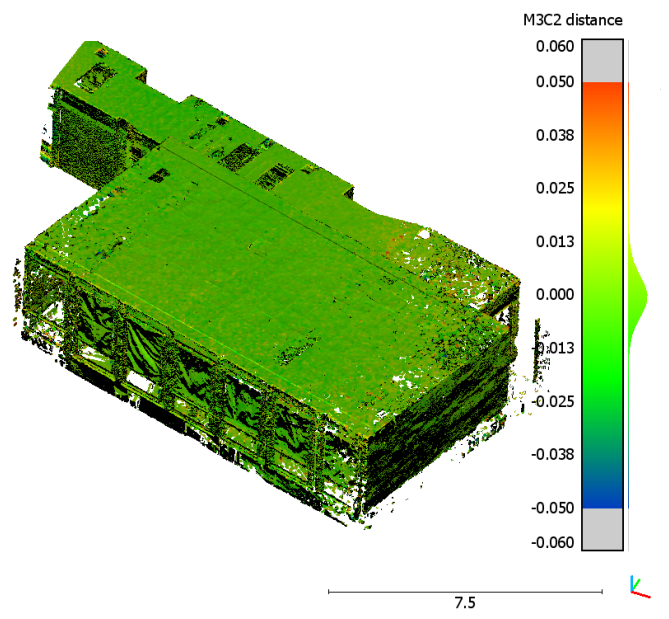

Figure 11. Representation of signed distances for loop 1.

\begin{tabular}{|c|c|}
\hline Settings & Values \\
\hline Number of static stations & 2 \\
Mean & $-0.0 \mathrm{~cm}$ \\
Standard deviation & $0.9 \mathrm{~cm}$ \\
Median & $-0.0 \mathrm{~cm}$ \\
MAD & $-0.7 \mathrm{~cm}$ \\
Skewness & -0.14 \\
Kurtosis & 9.8 \\
Distances between -3 and $3 \mathrm{~cm}$ & $99.8 \%$ \\
\hline
\end{tabular}

Table 9. Results for the loop 1.

The results offer a good perspective regarding the registration of an isolated room acquired with MMS on two SLS stations. A standard deviation of $9 \mathrm{~mm}$ and a percentage distance between -3 and $3 \mathrm{~cm}$ proves that the methodology is promising.

\subsubsection{Second and third room (Loop 2):}

For this last test two parts acquired with the same loop of MMS and 6 SLS stations are used as shown in Figure 2(b). The results of the comparison with the ground truth are illustrated in Figure 12 and the calculated parameters are listed in Table 10.

The room 1 presented in Figure 12 appears slightly bluish, showing that the deviations at this point are higher. This can be explained by the fact that the room 1 was scanned after the room 2 , thus increasing the error on the SLAM drift due to the duration of the acquisition. 


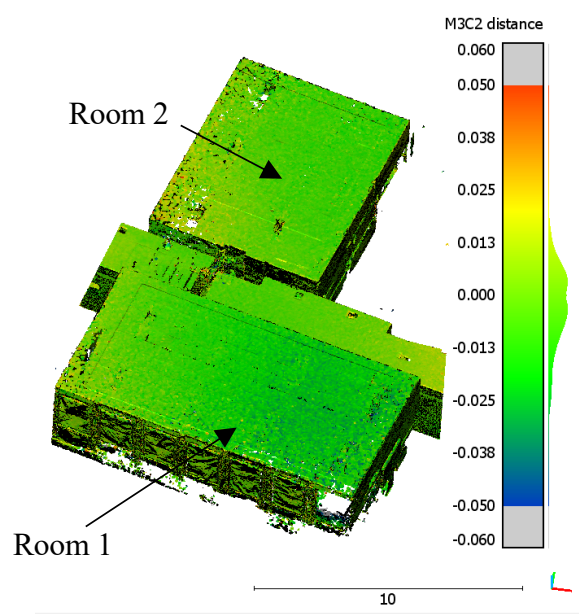

Figure 12. Representation of M3C2 signed distances for loop 2.

\begin{tabular}{|c|c|}
\hline Settings & Values \\
\hline Number of static stations & 3 \\
Mean & $-0.2 \mathrm{~cm}$ \\
Standard deviation & $1.1 \mathrm{~cm}$ \\
Median & $-0.2 \mathrm{~cm}$ \\
MAD & $-1.0 \mathrm{~cm}$ \\
Skewness & -0.107 \\
Kurtosis & 4.87 \\
Distances between -3 and $3 \mathrm{~cm}$ & $99.0 \%$ \\
\hline
\end{tabular}

Table 10. Results of loop 2 associated.

The results on both parts are satisfactory, with $99 \%$ of distances between -3 and $3 \mathrm{~cm}$. This means that the registration is homogeneous between the two parts.

\section{CONCLUSION AND PERSPECTIVES}

The study presented in this paper aimed to assess the coherence of merging point clouds acquired with static scanners and point clouds acquired with mobile mapping systems based on SLAM. These experiments led to the development of an acquisition methodology and some recommendations based on them.

For the horizontal loop, as expected, the reduction of the number of static stations reduces the accuracy of the registration (of MMS on SLS). However, the loss of accuracy depends on the length of the loop, the complexity of the location and how the rooms are cluttered. Segmenting the mobile point cloud into sub-clouds allows the drift of the instrument to be spread over the whole loop but does not correct it totally. Subsequently, the operation was repeated on a vertical loop of a stairwell. A compression effect of the mobile point cloud has been observed and a solution to distribute the error regularly along the trajectory has been proposed.

Furthermore, the reduction of static stations was studied, and it has been shown that keeping only a few but adequately placed static stations enables to use the handheld scanner more intensively with an acceptable loss of accuracy.

Based on these first experiments, a methodology combining the two systems is proposed for each of the three configurations: 1) for acquisitions in a linear corridor, one static scanner station can be kept in each corner and at each position where the mobile cloud might be segmented; 2) for a vertical acquisition such as in stairwells, one static station per landing should be kept or at least at each extremity of the stairwell. For both solutions, it remains necessary to control the quality of the registration between mobile and static clouds, as well as between the segments produced with the mobile point cloud.

Finally, the acquisition of an individual room can be done by positioning static stations in the corridor. The mobile acquisition is performed inside the room with an overlap in the corridor. At least two SLS stations have to be along the trajectory.

Since the results depend on the accuracy of the devices under study, it would be interesting to repeat these experiments with a more accurate mobile mapping system. In order to apply the recommendations proposed after this study, a large-scale acquisition methodology will be considered in a future work. A survey of the whole building will be carried out by scanning the corridors with a static system and the rooms connected to the corridor with a mobile mapping system.

\section{REFERENCES}

Lachat, E., Landes, T. and Grussenmeyer, P., 2018. Comparison of point cloud registration algorithms for better result assessment - towards an open-source solution, Int. Arch. Photogramm., Remote Sens. Spatial Inf. Sci., XLII-2, pp. 551-558. doi: 10.5194/isprs-archives-XLII-2-551-2018.

Lague, D., Brodu, N. and Leroux, J., 2013. Accurate 3D comparison of complex topography with terrestrial laser scanner: Application to the Rangitikei canyon (N-Z), ISPRS Journal of Photogrammetry and Remote Sensing, 82, pp. 10-26. doi: 10.1016/j.isprsjprs.2013.04.009.

Nocerino E, Menna F, Remondino F, Isabella I, RodríguezGonzálvez P., 2017. Investigation of indoor and outdoor performance of two portable mobile mapping systems, in Remondino, F. and Shortis, M. R. (eds). SPIE Optical Metrology, Munich, Germany, 16 pages, doi: 10.1117/12.2270761.

Previtali, M., Brumana, R. and Banfi, F., 2020. Existing infrastructure cost effective informative modeling with multisource sensed data: TLS, MMS and photogrammetry, Applied Geomatics, pp.20. doi: 10.1007/s12518-020-00326-3.

Salgues, H., Macher, H. and Landes, T., 2020. Evaluation of mobile mapping systems for indoor surveys, Int. Arch. Photogramm., Remote Sens. Spatial Inf. Sci., XLIV-4/W1-2020, pp. 119-125. doi: 10.5194/isprs-archives-XLIV-4-W1-2020$119-2020$

Toschi, I, P. Rodríguez-Gonzálvez, F. Remondino, S. Minto, S. Orlandini, A. Fuller, 2015. Accuracy evaluation of a mobile mapping system with advances statistical methods, Int. Arch. Photogramm., Remote Sens. Spatial Inf. Sci., XL-5/W4, pp. 245253. doi: 10.5194/isprsarchives-XL-5-W4-245-2015. 